\title{
Optimized Expression, Purification, and Rapid Detection of Recombinant Influenza Nucleoproteins Expressed in Sf9 Insect Cells
}

\author{
Sung-Jin Yoon ${ }^{1 \dagger}$, Young-Jun Park ${ }^{1,2 \dagger}$, Hyun Ju Kim ${ }^{3}$, Jinwoo Jang ${ }^{4}$, Sang Jun Lee ${ }^{3}$, Sunwoo Koo ${ }^{5 *}$, and \\ Moo-Seung Lee ${ }^{2,6 *}$ \\ ${ }^{1}$ Metabolic Regulation Research Center, Korea Research Institute of Bioscience and Biotechnology, Daejeon 34141, Republic of Korea \\ ${ }^{2}$ Department of Biomolecular Science, KRIBB School of Bioscience, Korea University of Science and Technology (UST), Daejeon 34113, \\ Republic of Korea \\ ${ }^{3}$ Department of Systems Biotechnology, Chung-Ang University, Anseong 17546, Republic of Korea \\ ${ }^{4}$ RED Center, Sugentech Inc., Daejeon 34025, Republic of Korea \\ ${ }^{5}$ Department of Neuroscience and Experimental Therapeutics, College of Medicine, Texas AEM University, 8447 Riverside Pkwy, Bryan, TX \\ 77807, USA \\ ${ }^{6}$ Infectious Disease Research Center, Korea Research Institute of Bioscience and Biotechnology, Daejeon 34141, Republic of Korea
}

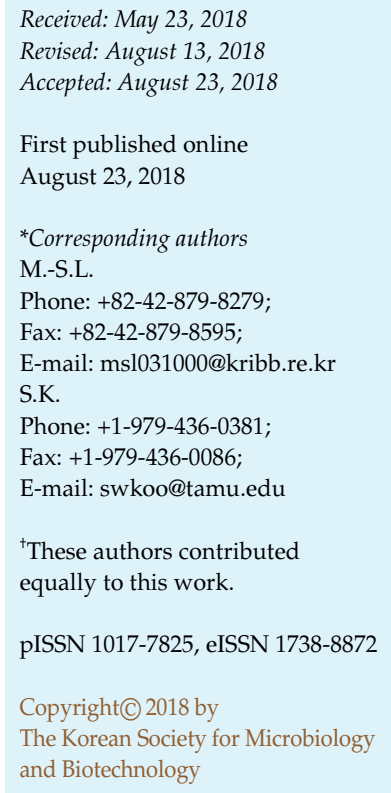

Accurate and rapid diagnosis of influenza infection is essential to enable early antiviral treatment and reduce the mortality associated with seasonal and epidemic infections. Immunochromatography is one of the most common methods used for the diagnosis of seasonal human influenza; however, it is less effective in diagnosing pandemic influenza virus. Currently, rapid diagnostic kits for pandemic influenza virus rely on the detection of nucleoprotein (NP) or hemagglutinin (HA). NP detection shows higher specificity and is more sensitive than HA detection. In this study, we time-dependently screened expression conditions, and herein report optimal conditions for the expression of recombinant nucleoprotein (rNP), which was $48 \mathrm{~h}$ after infection. In addition, we report the use of the expressed rNP in a rapid influenza diagnostic test (SGT i-flex Influenza A\&B Test). We constructed expression vectors that synthesized rNP (antigen) of influenza A and B in insect cells (Sf9 cells), employed the purified rNP to the immunoassay test kit, and clearly distinguished NPs of influenza A and influenza B using this rapid influenza diagnostic kit. This approach may improve the development of rapid test kits for influenza using NP.

Keywords: Influenza, rapid detection test, influenza nucleoprotein, Sf9 cells

\section{Introduction}

Influenza is an acute respiratory infection caused by influenza viruses of the Orthomyxoviridae family that can be classified on the basis of the composition of internal glycoproteins, nucleoprotein (NP), and matrix protein into subtypes: influenza virus A, influenza virus B, and influenza virus $C$. Types $A$ and $B$ are responsible for seasonal epidemics. Avian influenza A virus infection has been reported in humans, but it is not common [1]. The majority of cases of human influenza are clinically diagnosed, but definitive diagnosis requires the use of appropriate laboratory tests.

A number of diagnostic techniques are available for diagnosis of influenza infection in humans such as serology, immunochromatography-based rapid influenza diagnostic tests (RIDTs), and RNA detection by reverse transcription (RT)-PCR [2]. Conventional methods for detecting influenza virus have identified viral proteins by specific antibody staining or by hemadsorption of human erythrocytes in which the virus has propagated. The hemagglutinationinhibition assay (HAI) is a commonly used serological 
assay that determines the presence of antiviral antibodies in a patient's serum that inhibit hemagglutination by the virus in vitro [3]. The HAI is simple, inexpensive, and sensitive, but is limited in its ability to precisely diagnose subtypes of influenza virus [3]. Other common serological assays are based on the enzyme-linked immunosorbent assay (ELISA), and are available in either microtiter-plate or paper-strip formats, enabling rapid diagnosis [2]. Over the past decade, antiviral agents have been prescribed as first-line treatments for influenza patients. As these agents require administration within $72 \mathrm{~h}$ of the onset of fever, rapid diagnosis is necessary. RIDTs are antigen-based tests that can be completed in less than $30 \mathrm{~min}$. These tests have demonstrated variable performance for the diagnosis of seasonal influenza infections with sensitivities ranging from $20.6 \%$ to $70.4 \%$ [4]. PCR-based assays are the most reliable techniques for diagnosis of influenza infections and take $\sim 4 \mathrm{~h}$ to complete. They demonstrate high sensitivity and specificity, but are more expensive than serological tests and RIDTs.

The genomes of influenza A and B viruses are composed of eight single-stranded RNA molecules of negative polarity that are encapsidated by viral NP. NP is an antigen protein that has been used for the development of anti-influenza drugs and vaccines [5]. In addition, an anti-NP antibody specific to NP shows higher specificity and sensitivity than anti-hemagglutinin (HA) for the detection of influenza virus [6]. Because NP is antigenically conserved, it is a promising candidate for the development of assays for diagnosis of influenza virus infection [7]. An anti-NP MAb permits the rapid diagnosis of influenza virus (e.g., pandemic influenza H1N1) infection and shows higher sensitivity and specificity for pandemic H1N1 than for seasonal influenza A and B viruses [8]. The newly-developed pandemic diagnosis kit using anti-NP MAb detects seasonal influenza virus A/B, and has the potential to distinguish pandemic influenza viruses from seasonal influenza A and B viruses [9]. NP has been expressed using recombinant DNA methodology [7, 10]. In this study, we screened the expression of influenza virus NP using a recombinant DNA expression vector in a time-dependent manner, and we also examined the use of the NP in a rapid diagnostic test kit.

\section{Materials and Methods}

\section{Construction of NP Transfer Vector and Bacmid}

Cassettes for NP expression in insect cells contained the NP genes of influenza A/reassortant/NYMC X-179 (H1N1) virus or influenza B/Brisbane/60/2008 virus. These cassettes, which contained His-tag at the C-terminus of NP were cloned into pFastBac by double digestion with $\mathrm{XhoI}$ and BamHI restriction enzymes to construct a transfer vector, NP-His-GFP-pFastBac1, which was then transformed into competent cells, K12 DH10B ${ }^{\mathrm{TM}}$ T1R (Thermo Fisher Scientific, USA). Recombinant NP bacmids were generated using the Bac-to-Bac Baculovirus expression system (Thermo Fisher Scientific, USA). Briefly, the donor vector NP-HisGFP-pFastBac1 containing either the NP gene of influenza A/ reassortant/NYMC X-179 virus (pFastBac1-ANP) or influenza B/ Brisbane/60/2008 virus (pFastBac1-BNP) was transformed into the DH10 Bac E. coli strain to create recombinant NP bacmids by site-specific recombination of the transfer vectors with parent bacmids. The resulting expression vectors were screened by PCR sequencing with M13 primers to verify the presence of NP bacmids. pUC/M13 Forward: 5'-CCCAGTCACGACGTTGTAAAACG-3' pUC/M13 Reverse: 5'-AGCGGATAACAATTTCACACAGG-3'

\section{Expression and Cell Culture}

Sf9 insect cells from Spodoptera frugiperda were cultured in suspension at $27^{\circ} \mathrm{C}$ in Sf-900 serum free medium (SFM; Thermo Fisher Scientific, USA) supplemented with $1 \%$ penicillin/ streptomycin (Sigma-Aldrich, USA). To produce baculovirus for NP expression, recombinant bacmids were mixed with cellfectin reagent in Sf-900 SFM without supplements or antibiotics according to the manufacturer's instructions. The transfected cells were then incubated at $27^{\circ} \mathrm{C}$ for $72 \mathrm{~h}$ with daily monitoring for cytopathic effect (CPE). Baculoviruses directing expression of NP from influenza A/reassortant/NYMC X-179 or B/Brisbane/60/2008 were harvested from Sf9 cell culture supernatant at $72 \mathrm{~h}$ posttransfection. Collected supernatant was cleared by centrifuging at 3,000 rpm for $5 \mathrm{~min}$ and transferred to fresh 15-ml tubes as the P1 viral stock. The P1 virus was amplified to generate high-titer P3 virus. Viable cell-density, cell viability, and cell size were determined for Sf9 cells infected with P3 viruses at viral dilutions of 1:100, 1:1000, and 1:10,000, or mock-infected.

\section{NP Purification}

To optimize expression of NP proteins in scaled-up 11 cultures, Sf9 cells were cultured in shaking flasks with orbital rotation at $125 \mathrm{rpm}$ at $27^{\circ} \mathrm{C}$ and infected with $\mathrm{P} 3$ baculoviral stock. After $72 \mathrm{~h}$, the infected cells were harvested by centrifugation at 3,000 rpm for $15 \mathrm{~min}$ and washed three times with phosphate buffered saline (PBS, pH 7.4). Cell pellets were lysed with lysis buffer (50 mM Tris-HCl, $150 \mathrm{mM} \mathrm{NaCl}, 1 \mathrm{mM}$ EDTA, 1\% Triton X-100, 1\% sodium deoxycholate, $0.1 \%$ SDS, and $1 \mathrm{mM}$ PMSF) in an ice bath with gentle shaking for $1 \mathrm{~h}$. The lysate was centrifuged at $15,000 \mathrm{rpm}$ for $45 \mathrm{~min}$ at $4^{\circ} \mathrm{C}$. Cleared supernatant, which contained recombinant NP, was applied to a His-Trap HP column (1ml, GE Healthcare) with a flow rate of $1 \mathrm{ml} / \mathrm{min}$. After washing the column with washing buffer (50 mM Tris-HCL, $20 \mathrm{mM}$ Imidazole, $300 \mathrm{mM} \mathrm{NaCl}, \mathrm{pH} 7.5$ ), bound NP was eluted with a linear gradient of $20-500 \mathrm{mM}$ imidazole in $20 \mathrm{mM}$ Tris, $500 \mathrm{mM} \mathrm{NaCl}$, and eluted NP was dialyzed against PBS for $16 \mathrm{~h}$. The purity of 
recombinant NP was evaluated by SDS-PAGE with Coomassie Blue staining, and by western blot analysis. The concentration of purified NP was determined with a BCA protein assay.

\section{SDS-PAGE and Western Blot Analysis}

Lysates prepared from cells infected with recombinant baculovirus, as well as purified NP were subjected to $10 \%$ SDSPAGE at $120 \mathrm{~V}$ for $60 \mathrm{~min}$. Proteins were then transferred using the iBlot dry-blotting system (Thermo Fisher Scientific, USA) for
6 min to a polyvinylidene difluoride (PVDF) membrane which was then blocked with $5 \%$ non-fat dry milk in PBS with $0.1 \%$ Tween 20 (PBST). The blocked membrane was incubated with His-tag primary antibody at 1:10,000 dilution in PBS for $1.5 \mathrm{~h}$ at room temperature, then washed three times in PBST, and incubated with HRP-conjugated secondary antibody at 1:10,000 dilution (Cell Signaling Technology, USA). Bands were visualized with the ECL Prime chemiluminescence system (GE Healthcare, UK) and Image Studio 5.2 software (LI-COR, USA).

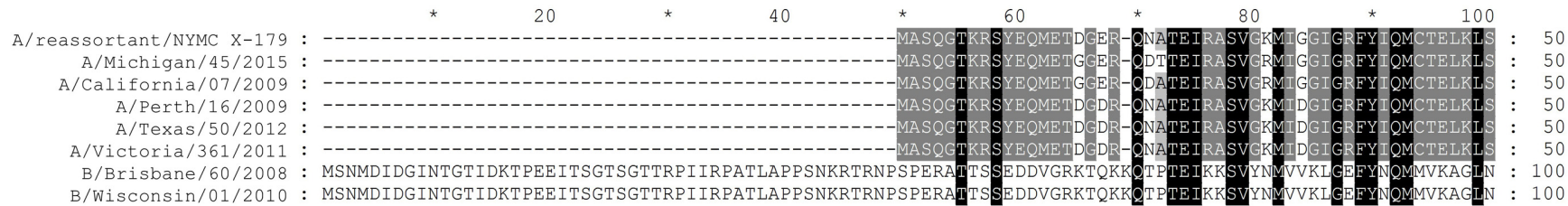

B/Wisconsin/01/2010: MSNMDIDGINTGTIDKTPEEITSGTSGTTRPIIRPATLAPPSNKRTRNPS PERATTSSEDDVGRKTQKKG
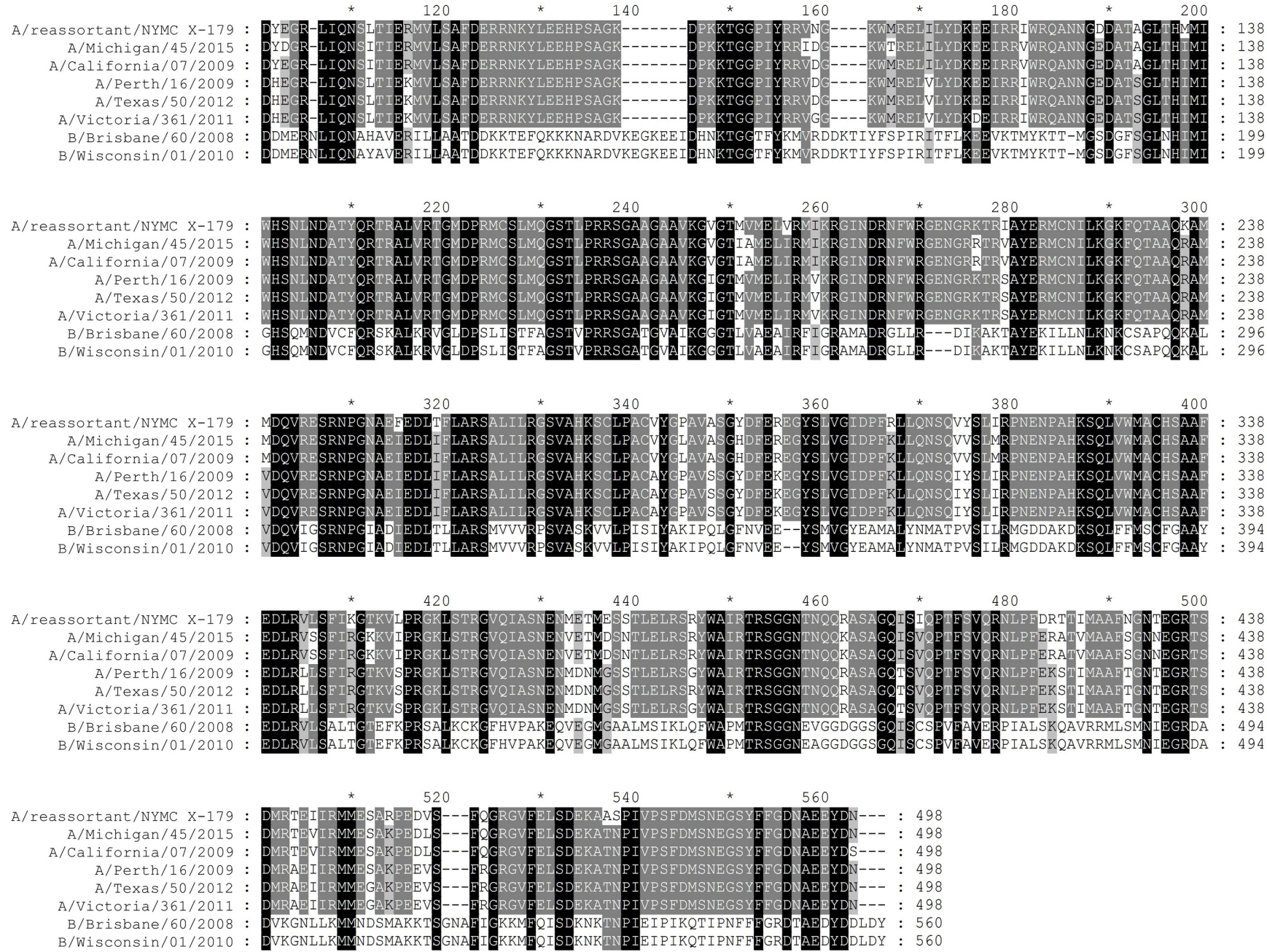

Fig. 1. Multiple sequence alignment of nucleocapsid proteins from influenza A and B viruses.

The shaded black and grey regions indicate identical amino acids and moderate conservation, respectively. Aligned sequences with NCBI accession numbers are as follows: A/reassortant/NYMC X-179 (California/07/2009 x NYMC X-157) (ADE29086.1), A/Michigan/45/2015 (APC60199.1), A/California/07/2009 (AUR34085.1), A/Perth/16/2009 (AHX37630.1), A/Texas/50/2012 (AIE52529.1), A/Victoria/361/2011 (AIE52624.1), B/Brisbane/60/2008 (AGK63064.1), and B/Wisconsin/01/2010 (AFH57958.1). 
Determination of the Sensitivity of a RIDT to Recombinant NP

The sensitivity of the RIDT (SGT i-flex Influenza A\&B Test, Sungentech Inc., South Korea) for the detection of purified NPs was assessed according to the manufacturer's instructions. Briefly, $50 \mu \mathrm{l}$ of NP $\left(0-10^{5} \mathrm{ng} / \mathrm{ml}\right)$ was mixed with $50 \mu \mathrm{l}$ of the sample buffer, and the mixture was applied to the sample well of the test cassette and incubated at room temperature for $10 \mathrm{~min}$ before the cassette was inserted into the portable immunoassay photometric analyzer (INCLIX, Sugentech Inc., Korea): signal on line 1 was the control; signal on line 2 was influenza B; and signal on line 3 was influenza A. The SGT Influenza A\&B test qualitatively detects influenza virus in the nasopharynx. Influenza virus swabs from nasopharynx are suspended in sample extracts, and the suspension is dispensed into the kit. Detection of influenza virus NP allows the diagnosis of influenza viruses through visual inspection and via antigen-antibody immunochromatography employing an NP specific antibody and gold. It is also possible to diagnose influenza A and B simultaneously and separately.

\section{Results}

\section{Sequence Alignments of NP from Influenza A and B Viruses}

We obtained eight NP sequences of influenza viruses from NCBI (six for influenza A, two for influenza B), which are recommended for use in influenza virus vaccine strains by the WHO (2011-2017). Percent sequence identity (identical residues/sequence length) was analyzed by multiple sequence alignments of the six NPs from influenza virus $A$ and two NPs from influenza B virus (Fig. 1). There was 89.6-99.6\% sequence identity among the six NPs from influenza A viruses, and $98.9 \%$ sequence identity between the two NPs of influenza B viruses. However, the sequence identities of NPs between influenza A and B viruses did not exceed $33.2 \%$. Thus, it was expected that full-length NPs expressed in influenza viruses A and B might have distinct properties as antigens in immunoassays.

\section{Expression of Recombinant Nucleoprotein (NP) from Influenza A or B in Sf9 Cells}

To construct the transfer vectors containing NP genes of influenza A/reassortant/NYMC X-179 (California/07/ 2009 x NYMC X-157; H1N1) (Fig. 2A) and B/Brisbane/60/ 2008 (Fig. 2B), codon-optimized, full-length NP genes fused with a 6-higtidine tag (His6) at the C-terminal region were assembled from synthetic oligonucleotides and cloned as a fusion cassette into the baculovirus transfer vector (pFastBac1) along with the polyhedrin promoter. An
A

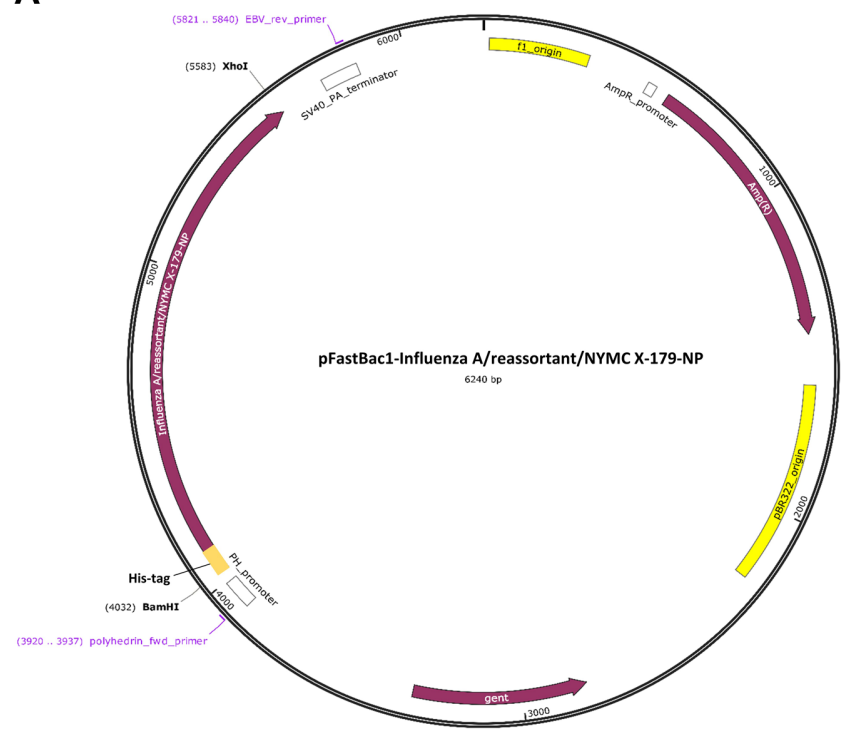

B

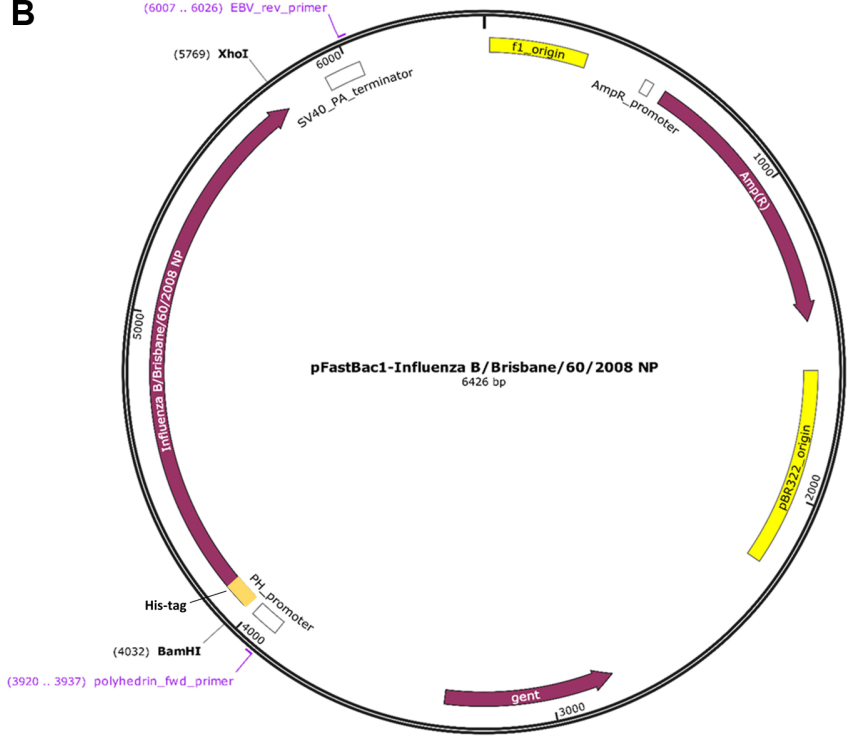

Fig. 2. Construction of donor vectors for expression of influenza $A / C a l i f o r n i a / 7 / 2009$ - recombinant nucleoprotein (rNP) or influenza B/Brisbane/60/2008-rNP.

(A) Map of the expression vector for His-tag-fused influenza A/California/7/2009-rNP. (B) Map of the expression vector for His-tag-fused influenza B/Brisbane/60/2008-rNP. The synthetic gene of interest (A/reassortant/NYMC X-179-NP or B/Brisbane/60/2008-NP) was assembled from synthetic oligonucleotides and/or PCR products. The fragment was inserted into pFastBac1. The recombinant plasmid DNA was purified from transformed Escherichia coli K12 DH10 T1R. Intact polyhedrin (PH) leader sequences may yield high levels of expression. The EK site is the recognition site for enterokinase cleavage between the affinity tag and NP. A His6 tag is located at the C-terminus of the gene of interest and ampicillin (AmpR) is used as a selection marker. 
A

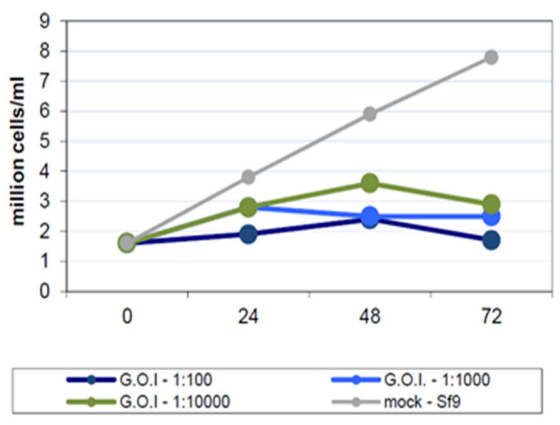

Influenza A NP bacmid-infected Sf9
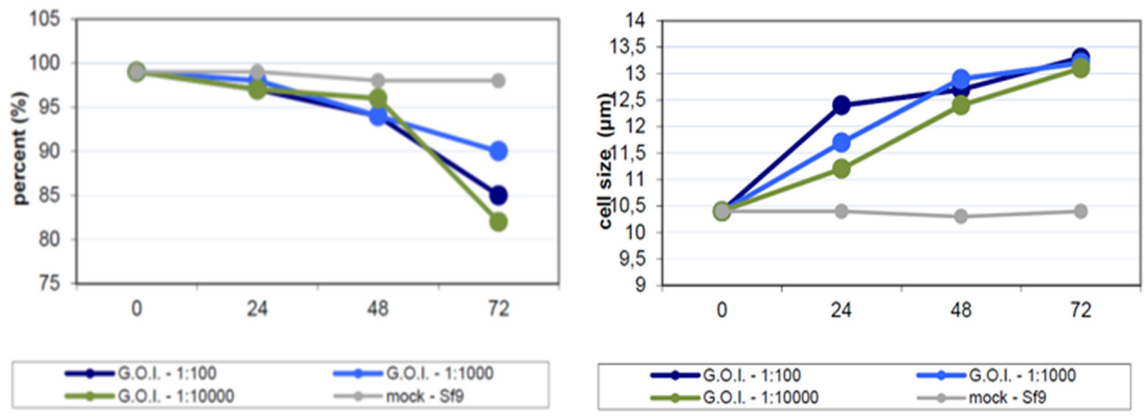

B

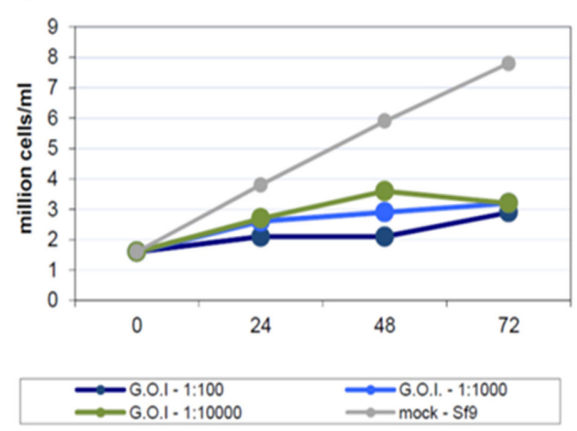

\section{Influenza B NP bacmid-infected Sf9}

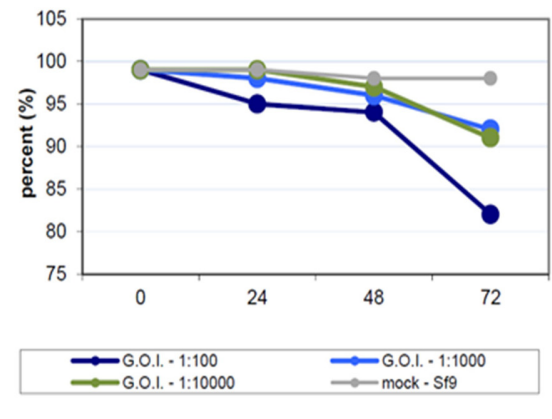

Fig. 3. Growth profiles of Sf9 insect cells expressing (A) recombinant influenza A/reassortant/NYMC X-179 (California/07/2009 x NYMC X-157; H1N1)-NP or (B) recombinant influenza B/Brisbane/60/2008-NP in 11 cultures.

The gene of interest (G.O.I.) in the form of a recombinant baculovirus, was introduced at 1:100, 1:1,000, and 1:10,000 dilutions, in addition to a mock-infection control. The viable cell density (left panel), cell viability (middle panels), and cell size (right panels) in NP-expressing Sf9 cells throughout $72 \mathrm{~h}$ of culture are indicated.

enterokinase recognition site was placed between His6-tag and the NP gene to facilitate tag cleavage. NP genes in pFastBac1 were transformed into the DH10 Bac strain to create the recombinant NP bacmid by recombination.

\section{Purification of Recombinant NP of Influenza A or B Expressed in Sf9 Cells}

Sf9 cell culture conditions are important for high level expression of NP. Sf9 cells were infected with influenza A NP bacmid (Fig. 3A) or influenza B NP bacmid (Fig. 3B) diluted 1:100, 1:1000, or 1:10,000, and then incubated for $72 \mathrm{~h}$ at $27^{\circ} \mathrm{C}$. Cell density, cell viability, and cell size were monitored every $24 \mathrm{~h}$ for a total of $72 \mathrm{~h}$, which showed that Sf9 cells infected with the influenza A/B NP bacmid that were cultured at $27^{\circ} \mathrm{C}$ for up to $72 \mathrm{~h}$ optimally expressed NP (Figs. 4A and 4B). Expression of total proteins in Sf9 cells infected with NP-expressing baculovirus was monitored by SDS-PAGE of cell lysates every $24 \mathrm{~h}$ for $72 \mathrm{~h}$ (Figs. $4 \mathrm{~A}$ and 4B). We used the baculovirus expression system instead of the bacterial protein expression system, because the former has several advantages over the latter [11, 12], namely it allows the expression of functional eukaryotic proteins with native folding; posttranslational modifications including phosphorylation, acylation and amidation; high level expression of the cloned gene; and large inserts. Expression of NP was verified with anti-His antibody that detects His-GFP (Histidine-GFP tag conjugated to C-terminus of NP) on western blots. NP from both influenza A virus (62 kDa) and influenza B virus (72 kDa) was expressed to a high level by $72 \mathrm{~h}$ in Sf9 cells (Figs. $4 \mathrm{~A}$ and $4 \mathrm{~B}$; lower panels). Notably, expression of influenza A NP was detected as early as at $24 \mathrm{~h}$ (at the highest viral concentration of 1:100 dilution), while influenza B NP was not. The purity of the purified NPs was demonstrated by Coomassie Bluestaining of SDS-PAGE gels, and the purified proteins were confirmed as recombinant NP by staining with anti-His 
A
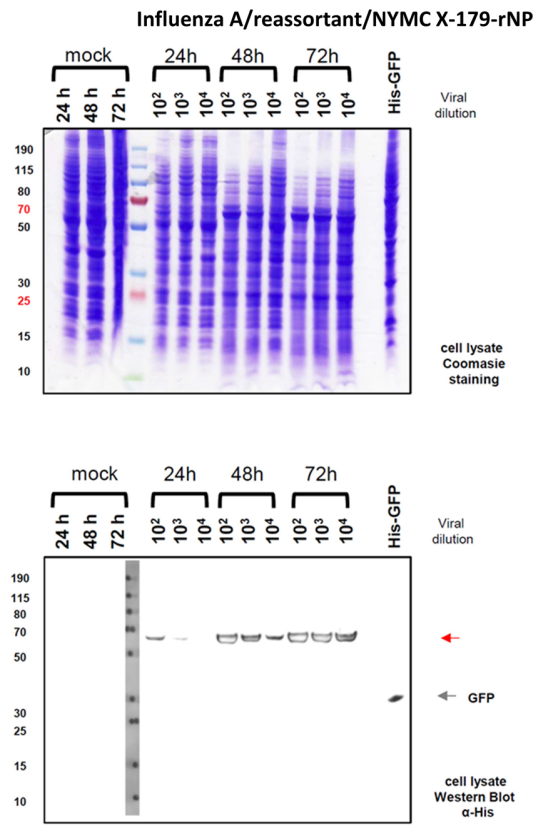

B
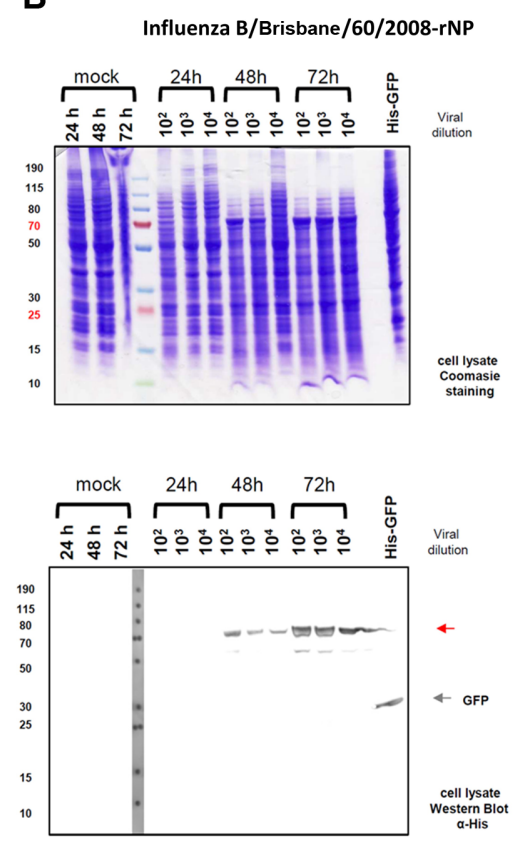
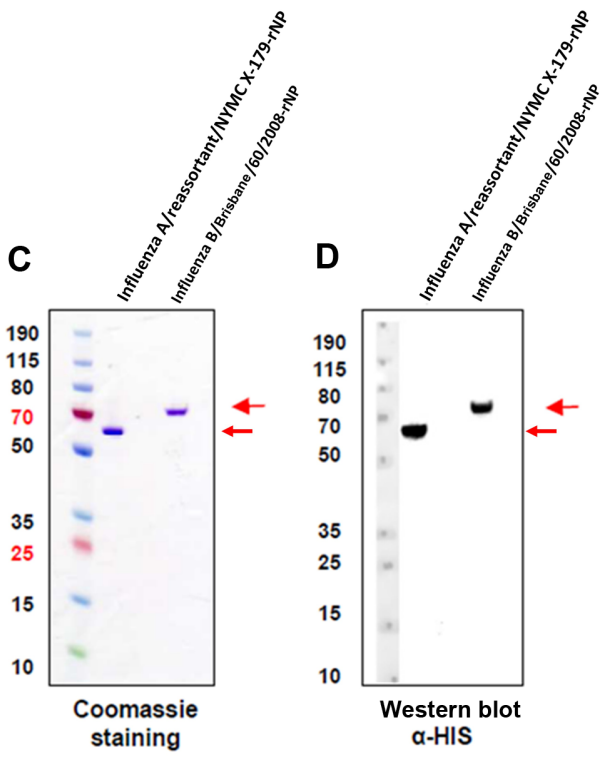

Fig. 4. Expression and purification of His-tag-fused recombinant nucleoprotein (rNP) in Sf9 insect cells.

(A) SDS-PAGE (upper panel) and western blotting (lower panel) of lysates from Sf9 cells with mock infection or infection with baculovirus directing expression of recombinant influenza A/reassortant/NYMC X-179-NP at 24-72 $\mathrm{h}$ post-infection at viral dilutions of 1:100, 1:1,000, and 1:10,000. (B) SDS-PAGE (upper panel) and western blotting (lower panel) of lysates from Sf9 cells with mock infection or infection with baculovirus directing expression of recombinant influenza B/Brisbane/60/2008-NP at 24-72 h post-infection at viral dilutions of 1:100, 1:1,000, and 1:10,000. (C) Purity of purified recombinant influenza A/reassortant/NYMC X-179-NP or B/Brisbane/60/2008-NP was determined by Coomassie Blue staining on SDS-PAGE. (D) Western blotting of purified recombinant NP proteins using an anti-His-tag antibody.

antibody on western blots (Figs. 4C and 4D). These results demonstrated that our system can be used to express efficiently and purify recombinant NP for the diagnosis of influenza A or B virus infection.

\section{Application of Recombinant NPs to a Rapid Diagnosis Test Kit}

The ability of an ELISA-based RIDT (SGT i-flex Influenza $\mathrm{A} \& \mathrm{~B}$ Test) to detect and discriminate between the influenza A
A

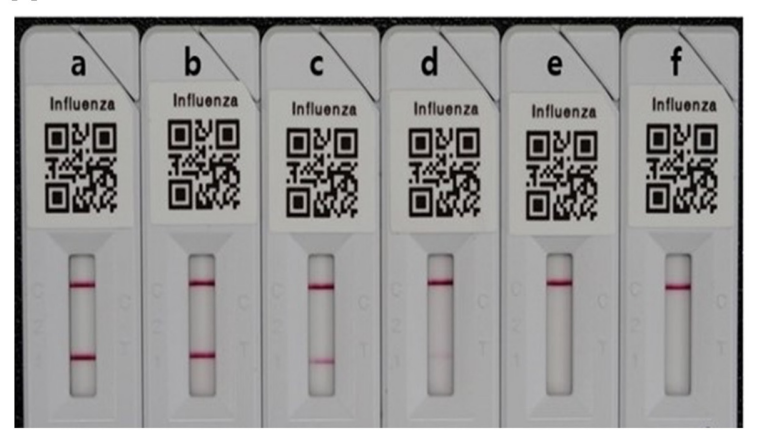

B

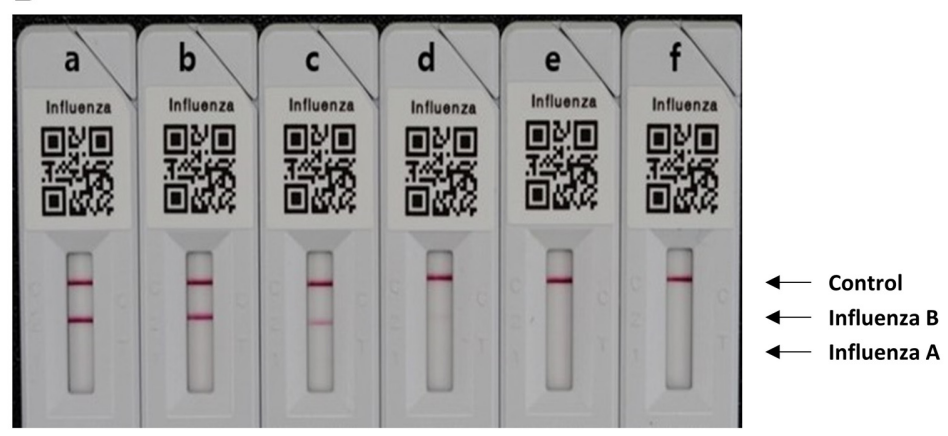

Fig. 5. Measurement of the limit of detection for recombinant influenza A or B nucleoprotein (NP) using a rapid influenza diagnostic test (SGT i-flex influenza A\&B Test).

(A) Antigenic activity of influenza virus A/reassortant/NYMC X-179 rNP at concentrations of (a) $10^{5} \mathrm{ng} / \mathrm{ml}$, (b) $10^{4} \mathrm{ng} / \mathrm{ml}$, (c) $10^{3} \mathrm{ng} / \mathrm{ml}$, (d) $10^{2} \mathrm{ng} / \mathrm{ml}$, (e) $10^{1} \mathrm{ng} / \mathrm{ml}$, and (f) $0 \mathrm{ng} / \mathrm{ml}$. (B) Antigenic activity of influenza virus B/Brisbane $/ 60 / 2008 \mathrm{NP}$ at concentrations of (a) $10^{5} \mathrm{ng} / \mathrm{ml}$, (b) $10^{4} \mathrm{ng} / \mathrm{ml}$, (c) $10^{3} \mathrm{ng} / \mathrm{ml}$, (d) $10^{2} \mathrm{ng} / \mathrm{ml}$, (e) $10^{1} \mathrm{ng} / \mathrm{ml}$, and (f) $0 \mathrm{ng} / \mathrm{ml}$. 
and B NPs was assessed. In this test, NPs bind specifically to antibodies attached to the surface of a membrane, and this binding is detected via a reaction that produces a colored band in one location for influenza A NP and in another for influenza B NP. The test was clearly able to discriminate between purified recombinant NP from influenza virus A/reassortant/NYMC X-179 (IAC, Fig. 5A) and from influenza virus B/Brisbane/60/2008 (IBB, Fig. 5B).

The limit of detection of the RIDT was similar for both $\mathrm{NPs}$, with faint bands produced with NP at $10^{2} \mathrm{ng} / \mathrm{ml}$ (Figs. 5A and 5B). Bose, M. et al. assessed 13 RIDTs and found a strong correlation between RIDT sensitivity and NP concentration, and most RIDTs were not sensitive at low concentrations of NPs including the California/07/2009 strain (mean concentration: $4.3 \mu \mathrm{g} / \mathrm{ml}$, range $0.7-13.4 \mu \mathrm{g} / \mathrm{ml}$ ) [13]. The approach described in this study could improve RIDT sensitivity for influenza virus diagnosis, help distinguish influenza A virus from influenza B virus infections, and aid in the future development of rapid diagnosis kits for influenza infection.

\section{Discussions}

Rapid and sensitive diagnostic tests for influenza virus infection are in high demand, as early diagnosis and treatment of influenza maximizes the efficacy of antiviral medicines. Here, we monitored the expression of NPs in the recombinant baculovirus expression system [10] to optimize NP expression. We constructed expression vectors that expressed strongly influenza A virus NP or influenza B virus NP, and optimized NP expression and its purification in Sf 9 cells. We then used the purified NPs in the rapid diagnosis test kit, which discriminated between recombinant NP from influenza A virus and that from influenza B virus. In addition, the expression of influenza A NP was detected at $24 \mathrm{~h}$ (only at high viral concentration, 1:100), $48 \mathrm{~h}$, and $72 \mathrm{~h}$ while influenza B NP was detected at $48 \mathrm{~h}$ and $72 \mathrm{~h}$ (Figs. 4A and 4B; lower panel). This indicated that our approach could detect infection by influenza virus based on different expression patterns over time. The specificity and sensitivity of NP in the RIDT was higher than that of hemagglutinin (HA) as an antigen [6]. Because there is a strong correlation between RIDT reactivity and the concentration of NP, measuring NP concentration is recommended for RIDT assessment [13]. Therefore, our approach may improve the reactivity of RIDT.

RIDTs and nucleic acid-based tests are commonly used for diagnosis of influenza because they are accurate, simple, and rapid. Although sensitivity varies between RIDTs, some such as BinaxNOW $A+B$, Quick Vue Influenza $A+B$, and Directigen EZ Flu $A+B$, have been FDA-approved and are used in many clinical virology laboratories. Lab-on-achip, based on complementation of RT-PCR with microfluidic technology, provides a powerful method for diagnosis of viral infection in humans, with results obtained within $30 \mathrm{~min}$ [14]. Next-generation sequencing (NGS) technology is rapidly developing and having an enormous impact on diagnostic medicine, such as with the identification of SNPs (single nucleotide polymorphisms) that cause antiviral resistance in seasonal influenza virus strains [15]. These modern methods are more rapid and sensitive than conventional diagnostic techniques, but are currently associated with high costs, which is a drawback that needs to be addressed.

Our approach involved the purification of NPs of influenza A and B viruses from insect cells to provide antigens for use in RIDTs. We improved the efficiency of protein expression and purification compared with previously reported results [7], and further demonstrated that influenza A virus NP and influenza B virus NP were clearly discriminated by the RIDT employing the recombinant NP.

\section{Acknowledgments}

This work was supported by the Korea Research Institute of Bioscience and Biotechnology (KRIBB) Research Initiative Program and by the Bio \& Medical Technology Development Program of the National Research Foundation (NRF), funded by the Korean government (MSIP) (NRF-2015M3A9E6028953 and 2016M3A9B6918675). This work was also supported by the Basic Research Program through the National Research Foundation of Korea (NRF), funded by the Ministry of Science, ICT \& Future Planning (grant number: 2017R1C1B1005137).

\section{Conflict of Interest}

The authors have no financial conflicts of interest to declare.

\section{References}

1. Wang F, Qi J, Bi Y, Zhang W, Wang M, Zhang B, et al. 2015. Adaptation of avian influenza A (H6N1) virus from avian to human receptor-binding preference. EMBO J. 34: 1661-1673.

2. Vemula SV, Zhao J, Liu J, Wang X, Biswas S, Hewlett I. 2016. Current approaches for diagnosis of influenza virus infections in humans. Viruses 8: 96.

3. Pedersen JC. 2014. Hemagglutination-inhibition assay for 
influenza virus subtype identification and the detection and quantitation of serum antibodies to influenza virus. Methods Mol. Biol. 1161: 11-25.

4. Nitsch-Osuch A, Wozniak-Kosek A, Brydak LB. 2012. Accuracy of rapid influenza diagnostic test and immunofluorescence assay compared to real time RT-PCR in children with influenza A(H1N1)pdm09 infection. Postepy Hig. Med. Dosw. (Online) 66: 752-757.

5. Wang W, Huang B, Jiang T, Wang X, Qi X, Tan W, et al. 2014. Maximal immune response and cross protection by influenza virus nucleoprotein derived from E. coli using an optimized formulation. Virology 468-470: 265-273.

6. Mizuike R, Sasaki T, Baba K, Iwamoto H, Shibai Y, Kosaka M, et al. 2011. Development of two types of rapid diagnostic test kits to detect the hemagglutinin or nucleoprotein of the swine-origin pandemic influenza A virus H1N1. Clin. Vaccine Immunol. 18: 494-499.

7. Rota PA, Black RA, De BK, Harmon MW, Kendal AP. 1990. Expression of influenza A and B virus nucleoprotein antigens in baculovirus. J. Gen. Virol. 71(Pt 7): 1545-1554.

8. Miyoshi-Akiyama T, Narahara K, Mori S, Kitajima H, Kase T, Morikawa S, et al. 2010. Development of an immunochromatographic assay specifically detecting pandemic H1N1 (2009) influenza virus. J. Clin. Microbiol. 48: 703-708.

9. Choi YJ, Kim HJ, Park JS, Oh MH, Nam HS, Kim YB, et al.
2010. Evaluation of new rapid antigen test for detection of pandemic influenza A/H1N1 2009 virus. J. Clin. Microbiol. 48: 2260-2262.

10. Kost TA, Condreay JP. 1999. Recombinant baculoviruses as expression vectors for insect and mammalian cells. Curr. Opin. Biotechnol. 10: 428-433.

11. Kidd IM, Emery VC. 1993. The use of baculoviruses as expression vectors. Appl. Biochem. Biotechnol. 42: 137-159.

12. Hoss A, Moarefi I, Scheidtmann KH, Cisek LJ, Corden JL, Dornreiter I, et al. 1990. Altered phosphorylation pattern of simian virus $40 \mathrm{~T}$ antigen expressed in insect cells by using a baculovirus vector. J. Virol. 64: 4799-4807.

13. Bose ME, Sasman A, Mei H, McCaul KC, Kramp WJ, Chen LM, et al. 2014. Analytical reactivity of 13 commercially available rapid influenza diagnostic tests with $\mathrm{H} 3 \mathrm{~N} 2 \mathrm{v}$ and recently circulating influenza viruses. Influenza Other Respir. Viruses 8: 474-481.

14. Ferguson BS, Buchsbaum SF, Wu TT, Hsieh K, Xiao Y, Sun R, et al. 2011. Genetic analysis of H1N1 influenza virus from throat swab samples in a microfluidic system for point-ofcare diagnostics. J. Am. Chem. Soc. 133: 9129-9135.

15. Levine M, Sheu TG, Gubareva LV, Mishin VP. 2011. Detection of hemagglutinin variants of the pandemic influenza A (H1N1) 2009 virus by pyrosequencing. J. Clin. Microbiol. 49: 1307-1312. 